\title{
Diffusion tensor imaging detects chronic microstructural changes in white and gray matter after traumatic brain injury in rat
}

\section{OPEN ACCESS}

Edited by:

Satrajit S. Ghosh,

Massachusetts Institute of

Technology, USA

Reviewed by:

Demian Wassermann,

INRIA Sophia Antipolis, France

Maxime Descoteaux,

Université de Sherbrooke, Canada

${ }^{*}$ Correspondence:

Alejandra Sierra,

A. I. Virtanen Institute for Molecular Sciences, University of Eastern

Finland, PO Box 1627,

Neulaniementie 2, Fl-70211 Kuopio,

Finland

alejandra.sierralopez@uef.fi

${ }^{\dagger}$ Shared first authorship.

Specialty section:

This article was submitted to Brain Imaging Methods,

a section of the journal

Frontiers in Neuroscience

Received: 09 January 2015

Accepted: 27 March 2015

Published: 22 April 2015

Citation:

Laitinen T, Sierra A, Bolkvadze T, Pitkänen A and Gröhn O (2015)

Diffusion tensor imaging detects

chronic microstructural changes in white and gray matter after traumatic brain injury in rat.

Front. Neurosci. 9:128.

doi: 10.3389/fnins.2015.00128

\author{
Teemu Laitinen ${ }^{1+}$, Alejandra Sierra ${ }^{1 * t}$, Tamuna Bolkvadze ${ }^{1}$, Asla Pitkänen ${ }^{1,2}$ and \\ Olli Gröhn ${ }^{1}$
}

'Department of Neurobiology, A. I. Virtanen Institute for Molecular Sciences, University of Eastern Finland, Kuopio, Finland,

${ }^{2}$ Department of Neurology, Kuopio University Hospital, Kuopio, Finland

Traumatic brain injury (TBI) is a major cause of disability and death in people of all ages worldwide. An initial brain injury caused by external mechanical forces triggers a cascade of tissue changes that lead to a wide spectrum of symptoms and disabilities, such as cognitive deficits, mood or anxiety disorders, motor impairments, chronic pain, and epilepsy. We investigated the detectability of secondary injury at a chronic time-point using ex vivo diffusion tensor imaging (DTI) in a rat model of TBI, lateral fluid percussion (LFP) injury. Our analysis of ex vivo DTI data revealed persistent microstructural tissue changes in white matter tracts, such as the splenium of the corpus callosum, angular bundle, and internal capsule. Histologic examination revealed mainly loss of myelinated axons and/or iron accumulation. Gray matter areas in the thalamus exhibited an increase in fractional anisotropy associated with neurodegeneration, myelinated fiber loss, and/or calcifications at the chronic phase. In addition, we examined whether these changes could also be detected with in vivo settings at the same chronic time-point. Our results provide insight into DTI detection of microstructural changes in the chronic phase of TBI, and elucidate how these changes correlate with cellular level alterations.

Keywords: diffusion tensor imaging, traumatic brain injury, microstructure, animal models, histology

\section{Introduction}

Traumatic brain injury (TBI) is a major cause of disability and death in people of all ages worldwide (Hyder et al., 2007). TBI occurs by external mechanical forces, such as falls, vehicle accidents, violence, and sports (Maas et al., 2008). The location, type, and severity of the initial injury determine the prognosis, which varies from patient to patient. This heterogeneity leads to a wide spectrum of symptoms and disabilities, such as cognitive deficits, mood or anxiety disorders, motor impairments, chronic pain, and epilepsy. After brain injury, proper early evaluation and follow-up of the progression are crucial to improve diagnosis, management, and treatment (Maas et al., 2008).

Abbreviations: $\mathrm{ab}$, angular bundle; $\mathrm{ac}$, anterior commissure; $\mathrm{cc}_{\mathrm{g}}$, genu of the corpus callosum; $\mathrm{cc}_{\mathrm{b}}$, body of the corpus callosum; $c_{s}$, splenium of the corpus callosum; $\mathrm{D}_{\|}$, axial diffusivity; $\mathrm{D}_{\perp}$, radial diffusivity; $\mathrm{FA}$, fractional anisotropy; ic, internal capsule; LD, laterodorsal thalamic nucleus; LFP, lateral fluid percussion; MD, mean diffusivity; MRI, magnetic resonance imaging; ROI, region of interest; TBI, traumatic brain injury; VPM, ventroposteromedial nucleus; VPL, ventroposterolateral nucleus. 
The initial assessment of primary lesions caused by the initial injury, such as hemorrhage, tissue deformation, and edema, is currently performed using computed tomography (CT) (Marshall et al., 1991), which is the gold standard for acute examination of patients after head injury. Magnetic resonance imaging (MRI) is increasingly used in subacute and chronic phases, due to the versatile MRI contrasts available to gain better insight into the secondary damage (Gallagher et al., 2007; Aquino et al., 2015). Advanced MRI techniques, such as diffusion tensor imaging (DTI), were developed for more accurate evaluation of secondary damage (Basser et al., 1994; Basser and Pierpaoli, 1996). DTI measures the magnitude and directionality of water diffusion, which depends on the tissue microstructure (Mori and Zhang, 2006). Furthermore, under pathologic conditions, DTI provides complementary information regarding tissue integrity (Song et al., 2002; Budde et al., 2011).

Previous studies of TBI patients used DTI to evaluate progressive white matter injury, which is potentially linked to poor outcome or recovery in those patients (Sidaros et al., 2008; Kumar et al., 2009). A recent study using DTI and statistical image analysis, tract-based spatial statistics (TBSS), revealed that several brain regions are affected in patients with TBI (Kinnunen et al., 2011). Trauma-induced structural alterations in the brain are detected by DTI with much higher sensitivity than conventional anatomic imaging, but little is known about the histopathology underlying these alterations.

Some previous experimental TBI studies investigated alterations in DTI parameters in rodents, followed by histologic examination (Mac Donald et al., 2007a,b; Budde et al., 2011). These studies, however, focused mainly on acute and subacute time points after injury, and the consequences of slowly progressing secondary damage to tissue microstructure have not been evaluated (Mac Donald et al., 2007a,b; Budde et al., 2011; Hylin et al., 2013).

In the present study, we investigated the detectability of secondary injury at a chronic time-point in a rat model of TBI, lateral fluid percussion (LFP) injury (McIntosh et al., 1989), using ex vivo DTI. Extensive histologic examination was performed to associate the imaging findings with tissue alterations at the cellular level. Additionally, we performed an in vivo DTI experiment at the same chronic time-point to test whether the changes could potentially serve as non-invasive markers of cellular level alterations in chronic TBI.

\section{Materials and Methods}

\section{Induction of Traumatic Brain Injury by Lateral Fluid Percussion}

TBI was induced in adult male Sprague-Dawley rats $(n=$ 13, 10 weeks old, weight 300-350 g, Harlan Netherlands B.V., Horst, Netherlands) by LFP injury, as described previously (Kharatishvili et al., 2006). Briefly, rats were anesthetized with a single intraperitoneal injection $(6 \mathrm{ml} / \mathrm{kg})$ of a mixture containing sodium pentobarbital (58 mg/kg), chloral hydrate $(60 \mathrm{mg} / \mathrm{kg})$, magnesium sulfate $(127.2 \mathrm{mg} / \mathrm{kg})$, propylene glycol $(42.8 \%)$, and absolute ethanol (11.6\%). A craniotomy ( $\varnothing 5 \mathrm{~mm}$ ) between bregma and lambda on the left skull convexity was then performed (anterior edge $2.0 \mathrm{~mm}$ posterior to the bregma; lateral edge adjacent to the left lateral ridge). LFP injury was induced by a transient (21-23 ms) fluid pulse impact against the exposed intact dura using a fluid-percussion device. The impact pressure was adjusted to induce a severe injury (3.26 $\pm 0.07 \mathrm{~atm})$. Shamoperated control animals $(n=7)$ underwent the same surgical procedures without the impact.

Following the procedures, the animals were housed in individual cages and maintained under a 12-h light/12-h dark cycle (lights on at 07:00 a.m., temperature $22 \pm 1^{\circ} \mathrm{C}$, air humidity 50$60 \%$ ) with free access to food and water. All animal procedures were approved by the Animal Care and Use Committee of the Provincial Government of Southern Finland and conducted in accordance with the guidelines set by the European Community Council Directives 86/609/EEC.

\section{Tissue Preparation}

Six months after TBI, 7 control rats and 8 rats with TBI were deeply anesthetized and transcardially perfused according to the following protocols. Approximately half of the animals (4 controls, $4 \mathrm{TBI}$ ) were perfused according to the Timm fixation protocol (Sloviter, 1982). Briefly, the animals were perfused with $0.37 \%$ sulfide solution $(30 \mathrm{ml} / \mathrm{min}$ ) for $10 \mathrm{~min}$, followed by $4 \%$ paraformaldehyde in $0.1 \mathrm{M}$ phosphate buffer, $\mathrm{pH} 7.4(30 \mathrm{ml} / \mathrm{min})$, $4^{\circ} \mathrm{C}$ for $10 \mathrm{~min}$. The brains were removed from the skull, postfixed in $4 \%$ paraformaldehyde in $0.1 \mathrm{M}$ phosphate buffer for $6 \mathrm{~h}$, and then washed in $0.9 \% \mathrm{NaCl}$ for at least $24 \mathrm{~h}$ before MRI. The remaining animals (3 controls, $4 \mathrm{TBI}$ ) were transcardially perfused using a $\mathrm{pH}$-shift fixation protocol (Savander et al., 1996). The rats were perfused with $0.9 \% \mathrm{NaCl}(30 \mathrm{ml} / \mathrm{min})$ for $2 \mathrm{~min}$, followed by $4 \%$ paraformaldehyde in $0.1 \mathrm{M}$ sodium acetate buffer $\left[\mathrm{NaC}_{2} \mathrm{H}_{3} \mathrm{O}_{2}, \mathrm{pH} 6.5(30 \mathrm{ml} / \mathrm{min}), 4^{\circ} \mathrm{C}\right]$ for $10 \mathrm{~min}$, and then with $4 \%$ paraformaldehyde in $0.1 \mathrm{M}$ sodium borate buffer $\left[\mathrm{Na}_{2} \mathrm{~B}_{4} \mathrm{O}_{7} \cdot 10 \mathrm{H}_{2} \mathrm{O}, \mathrm{pH} 9.5(30 \mathrm{ml} / \mathrm{min}), 4^{\circ} \mathrm{C}\right]$ for $15 \mathrm{~min}$. Before ex vivo DTI, the brains were removed from the skull, post-fixed in $0.1 \mathrm{M}$ sodium borate for $6 \mathrm{~h}$, and then washed in $0.9 \% \mathrm{NaCl}$ for at least $24 \mathrm{~h}$ before MRI. During the measurements, they were immersed in perfluoro polyether (Fomblin ${ }^{\circledR}$ LC08, Solvay Solexis, Milan, Italy) to avoid signal from the solution.

\section{Ex Vivo DTI}

For ex vivo DTI, brains from control rats and rats with TBI were scanned in a vertical $9.4 \mathrm{~T} / 89 \mathrm{~mm}$ magnet (Oxford Instruments PLC, Abingdon, UK) interfaced to a DirectDrive console (Varian Inc., Palo Alto, CA, USA) using a quadrature volume RFcoil (diameter $20 \mathrm{~mm}$; Rapid Biomedical GmbH, Rimpar, Germany) as the transmitter and receiver. Data were acquired using a three-dimensional (3D) spin echo sequence with two refocusing pulses $(T R=1 \mathrm{~s}, T E=60 \mathrm{~ms}$, data matrix $256 \times 74 \times 56$, FOV $29.3 \times 17 \times 12.8 \mathrm{~mm}^{3}$ ). To compensate for long-duration constant eddy currents, monopolar diffusion gradient pairs with opposite polarity for each pair were placed around each refocusing pulse. Six 3D images with diffusion weighting (diffusion time $=17 \mathrm{~ms}, b$-value $=1000 \mathrm{~s} / \mathrm{mm}^{2}$ ) in six uniformly distributed directions (Basser and Pierpaoli, 1998) and one image 
without diffusion weighting were obtained. Two averages were acquired, leading to a total scanning time of $16 \mathrm{~h} / \mathrm{brain}$. This type of protocol produces high signal-to-noise images, making accurate estimation of DTI metrics possible from only six orthogonal diffusion directions. The temperature variation of the sample during the DTI measurement period was less than $\pm 0.25^{\circ} \mathrm{C}$ in separate experiments under identical conditions.

\section{Histology to Verify the Ex Vivo DTI Findings Tissue Processing}

After ex vivo imaging, brains were washed in $0.9 \% \mathrm{NaCl}$ for at least $2 \mathrm{~h}$ at $4^{\circ} \mathrm{C}$ and then placed in a cryoprotective solution containing $20 \%$ glycerol in $0.02 \mathrm{M}$ potassium phosphate-buffered saline $(\mathrm{pH} 7.4)$ for $36 \mathrm{~h}$. The brains were then frozen in dry ice and stored at $-70^{\circ} \mathrm{C}$ until sectioning. The brains were sectioned in the coronal plane ( $30 \mu \mathrm{m}, 1$-in-5 series) using a sliding microtome. The first series of sections was stored in $10 \%$ formalin and the remaining series was stored in a cryoprotectant tissue-collecting solution (30\% ethylene glycol, $25 \%$ glycerol in $0.05 \mathrm{M}$ sodium phosphate buffer) at $-20^{\circ} \mathrm{C}$ until further processing.

\section{Staining for Assessment of Neurodegeneration, Gliosis, Myelination, Iron Deposits, and Calcifications} The first series of sections was stained with thionin (Nissl) to assess the cytoarchitectonics, gliosis, and neurodegeneration in different brain areas. The second series was stained with gold chloride solution to assess the myelinoarchitectonics, damage to myelinated axons, and also plasticity in myelinated pathways (Laitinen et al., 2010). Sections from the third series were stained with alizarin red to detect calcification, which occurs particularly in the thalamus (Mäkinen et al., 2008). Finally, selected sections were stained with Perl's method to confirm the presence of iron from post-TBI bleeds within the tissue.

The severity of neurodegeneration and loss of myelinated axons, and the presence of calcification and iron deposits were semi-quantitatively scored from 0 to 3 : none (score 0 ), mild (score 1), moderate (score 2), and severe (score 3 ) in rats with TBI as compared with controls (representative examples of scoring are shown in Figure 3). Finally, we calculated the overall damage score for each animal as the sum of individual scores based on each type of staining.

\section{In Vivo DTI}

We next assessed whether the histologically verified ex vivo DTI changes in the gray and white matter could also be detected using in vivo DTI. For this, TBI was induced in another group of male Sprague-Dawley rats ( $n=9,10$ weeks old, weight $300-350 \mathrm{~g}$ ) with LFP injury $(3.42 \pm 0.10 \mathrm{~atm})$. Sham-operated rats were used as controls $(n=3)$. Six months later, in vivo DTI was performed in a horizontal $9.4 \mathrm{~T} / 31 \mathrm{~cm}$ magnet interfaced to a Varian DirectDrive console (Varian Inc.). An actively decoupled transmitter volume RF-coil (diameter $72 \mathrm{~mm}$ ) -4 channel surface receiver RF-coil optimized for rat head (Rapid Biomedical GmbH) was used for the acquisitions. The animals were anesthetized with $1.4 \%$ isoflurane in $\mathrm{N}_{2} \mathrm{O} / \mathrm{O}_{2}(70 \% / 30 \%)$ and fixed in a stereotaxic holder. Normal body temperature was maintained with a heating pad. For data acquisition, we used a 3D-segmented spin-echo EPI with a navigator echo for phase correction, a Nyquist ghost minimization scheme similar to by van der Zwaag et al. (2009), and outer volume suppression $(T R=2 \mathrm{~s}, T E=45 \mathrm{~ms}, 2$ segments, data matrix $128 \times 64 \times 64$, FOV $25 \times 15 \times 15 \mathrm{~mm}^{3}, 1$ average). Six images with diffusion weighting applied to six uniformly distributed directions $(\triangle=16 \mathrm{~ms}, \delta=5 \mathrm{~ms}, b$-value $=$ $1000 \mathrm{~s} / \mathrm{mm}^{2}$ ) and one image without diffusion weighting were acquired for in vivo DTI (Basser and Pierpaoli, 1998). The total acquisition time for DTI data was $2 \mathrm{~h}$.

\section{Ex Vivo and In Vivo DTI Data Analysis}

Ex vivo DTI data were zero padded to $256 \times 148 \times 112$ point prior Fourier-transformation leading to an interpolated spatial resolution of $115 \times 115 \times 115 \mu \mathrm{m}^{3}$. In vivo DTI data were first corrected for Nyquist ghosting as described by van der Zwaag et al. (2009). Next, two consecutive k-space lines in the second phase encoding direction were added together to achieve a higher signal-to-noise ratio in the images, leading to a data matrix of $128 \times 64 \times 32$ and spatial resolution of $195 \times 234 \times 469 \mu \mathrm{m}^{3}$. All data were corrected for residual eddy current distortions with affine linear alignment of the diffusion-weighted images to the image without diffusion weighting with FMRIB's Linear Image Registration Tool (FLIRT) (Jenkinson and Smith, 2001; Jenkinson et al., 2002), which is included in FMRIB Software Library software (http://www.fmrib.ox.ac.uk/fsl/). Following eddy current corrections, maps of the diffusion tensor eigenvalues $\left(\lambda_{1}, \lambda_{2}, \lambda_{3}\right)$, trace of the diffusion tensor [trace(D)], and fractional anisotropy (FA), as well as directionally encoded color maps, where the color of each voxel is defined by the orientation of its diffusion tensors primary eigenvector $\left(\mathrm{V}_{1}\right)$ and the intensity of each voxel is proportional to FA (Pajevic and Pierpaoli, 1999), were created using DTI-Studio 2.40 (https://www.mristudio.org/). From the eigenvalue maps, maps of axial diffusivity $\left(D_{\|}=\lambda_{1}\right)$ showing the diffusivity parallel to the direction of the principal eigenvector and radial diffusivity $\left(D_{\perp}=\left(\lambda_{2}+\lambda_{3}\right) / 2\right)$ describing the diffusivity perpendicular to the principal eigenvector were created, and from the trace $(\mathrm{D})$-map a map of mean diffusivity $(\mathrm{MD}=\operatorname{trace}(\mathrm{D}) / 3)$ was determined using in-house built MATLAB (2010a, Mathworks, Natick, MA, USA) code and the region of interest (ROI) analysis was performed using an in-house built MATLAB code (http://aedes.uef.fi/).

Reliable co-registration of the brain with the atlas was not possible due to large variably-sized lesions and profound atrophy in the TBI brain. Therefore, ROIs were manually outlined based on directionally encoded color maps by one of the authors (AS.) with extensive experience in neuroanatomy, both in histologic preparations and MRI. Several consecutive slices were outlined on the coronal plane to cover the entire anatomic brain volume. The selected ROIs included the angular bundle (ab), anterior commissure $(\mathrm{ac})$, genu of the corpus callosum $\left(\mathrm{cc}_{\mathrm{g}}\right)$, body of the corpus callosum $\left(\mathrm{cc}_{\mathrm{b}}\right)$, splenium of the corpus callosum $\left(\mathrm{cc}_{\mathrm{s}}\right)$, internal capsule (ic), laterodorsal thalamic nuclei (LD), and a region comprising the ventroposteromedial (VPM), and ventroposterolateral (VPL) nuclei. The same ROIs were selected for analysis from both the ex vivo and in vivo data (Figure 1). 


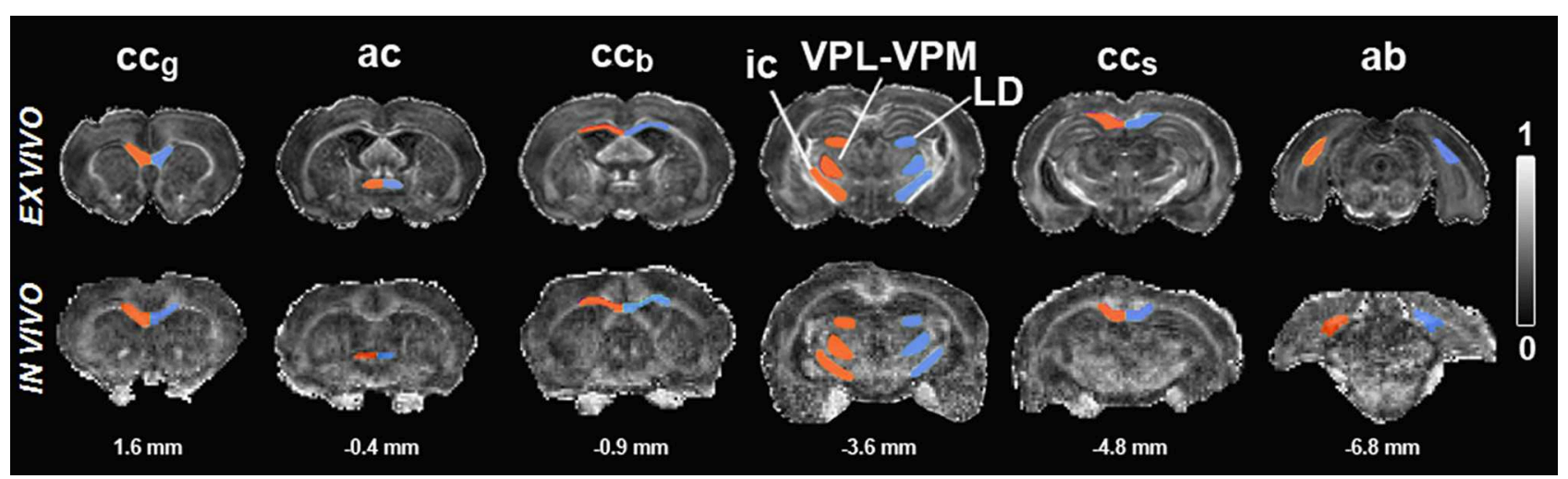

FIGURE 1 | Fractional anisotropy maps of sham-operated animals ex vivo (upper row) and in vivo (lower row). Regions of interest are outlined in red (ipsilateral) and blue (contralateral). Scale bar: 0 (black) to 1 (white). Abbreviations: ac, anterior commissure; $a b$, angular bundle; $c c_{b}$, body of the corpus callosum; $c c_{g}$, genu of corpus callosum; ic, internal capsule; $L D$, laterodorsal thalamic nuclei; $c c_{S}$, splenium of corpus callosum; VPL-VPM, ventroposteromedial (VPM), and ventroposterolateral (VPL) nuclei. Numbers indicated below the maps correspond to the distance from bregma.

\section{Histology from In Vivo Samples}

We also examined histologic sections from in vivo group to confirm that the neurodegeneration and loss of myelinated axons, as well as the presence of calcification and iron deposits, were comparable between the two groups.

\section{Statistical Analysis}

Data were analyzed using IBM SPSS 19.0 for Windows (SPSS Inc., Chicago, IL, USA). Data for controls and rats with TBI were compared using the Mann-Whitney $U$-test. Interhemispheric differences were compared using the Wilcoxon test. Results are expressed as mean \pm standard deviation (SD). A $p$-value of less than 0.05 was considered statistically significant.

\section{Results}

\section{Mortality and Location and Extent of Cortical Lesions after LFP Injury}

Mortality in the group of rats prepared for ex vivo study was $38 \%(5 / 13)$ and for the in vivo study, $44 \%\left(4 / 9 ; p=0.779, \chi^{2}\right.$ test). As assessed from histologic preparations, the location of the injury was consistent in all of the rats after TBI in both the ex vivo and in vivo groups: cortical lesions were observed between approximately -2 and $-6 \mathrm{~mm}$ from bregma.

\section{Abnormalities in White Matter Tracts after LFP Injury \\ Corpus Callosum}

As the severity and extent of cortical damage at different rostrocaudal levels varied remarkably between the animals, we assessed whether the damage to the corpus callosum varied accordingly. Histologic and DTI analyses were conducted separately in the genu $\left(\mathrm{cc}_{\mathrm{g}}\right)$, body $\left(\mathrm{cc}_{\mathrm{b}}\right)$, and splenium $\left(\mathrm{cc}_{\mathrm{s}}\right)$ of the corpus callosum (Figure 1).

\section{Genu of the Corpus Callosum}

There were no detectable abnormalities in DTI or histology (Tables 1-3).

\section{Body of the Corpus Callosum Ex vivo DTI}

The $\mathrm{cc}_{\mathrm{b}}$ showed no changes in any of the parameters analyzed, ipsilaterally or contralaterally (Table 1).

\section{Histology from ex vivo DTI samples}

In sections that were sampled from the level of the $\mathrm{cc}_{\mathrm{b}}$ analyzed in ex vivo DTI, we observed remarkable thinning of the structure, reflecting the loss of myelinated axons (Figures 2A-D). Myelin loss was extensive ipsilaterally in all the rats $(n=8)$, and loss was moderate to severe (score 2 or 3 ) in 6 of 8 animals (Table 2). Myelin loss was also observed contralaterally in 7 of 8 rats, and loss was moderate to severe in 2 of 8 animals (Table 2). Iron accumulation was observed ipsilaterally in 3 of 8 rats with TBI (Table 2; Figures 3A,B). Although overall thinning of the $\mathrm{cc}_{\mathrm{b}}$ was observed, the actual structure on the ipsilateral side remained similar to that on the contralateral side; myelinated axons run parallel along the tracts, which might explain the lack of significant findings in ex vivo DTI data.

\section{In vivo DTI}

Consistent with ex vivo data, none of the parameters analyzed showed any statistically significant changes (Table 3 ).

\section{Histology from in vivo DTI samples}

Histologic analysis revealed similar myelin loss and iron accumulation ipsilaterally as observed in ex vivo DTI samples from injured animals.

\section{Splenium of the Corpus Callosum \\ Ex vivo DTI}

The $\mathrm{cc}_{s}$ did not show changes in $\mathrm{FA}$ or $\mathrm{D}_{\perp}$, but $\mathrm{D}_{\|}$was higher ipsilaterally than contralaterally $(p<0.01)$ in rats after TBI (Table 1; Figures 2E,F). MD increased ipsilaterally compared with the contralateral side $(p<0.05$; Table 1$)$. 
TABLE 1 | Fractional anisotropy (FA), and axial $\left(D_{||}\right)$, radial $\left(D_{\perp}\right)$, and mean (MD) diffusivities from ipsilateral and contralateral hemispheres in controls $(n=7)$ and rats after TBI $(n=8)$ obtained using ex vivo DTI.

\begin{tabular}{|c|c|c|c|c|c|c|c|c|c|c|}
\hline \multicolumn{3}{|c|}{ Ex vivo } & \multicolumn{2}{|c|}{ FA } & \multicolumn{2}{|c|}{$D_{\|}\left(10^{-3} \mathrm{~mm}^{2} / \mathrm{s}\right)$} & \multicolumn{2}{|c|}{$D_{\perp}\left(10^{-3} \mathrm{~mm}^{2} / \mathrm{s}\right)$} & \multicolumn{2}{|c|}{$\mathrm{MD}\left(10^{-3} \mathrm{~mm}^{2} / \mathrm{s}\right)$} \\
\hline & & & Control & TBI & Control & TBI & Control & TBI & Control & TBI \\
\hline \multirow{8}{*}{$\begin{array}{l}\frac{\widetilde{\sigma}}{\bar{\Phi}} \\
\frac{\widetilde{\sigma}}{\bar{\omega}} \\
\underline{\underline{0}}\end{array}$} & White & $\mathrm{CC}_{\mathrm{g}}$ & $0.78 \pm 0.06$ & $0.81 \pm 0.05$ & $1.26 \pm 0.18$ & $1.22 \pm 0.18$ & $0.24 \pm 0.05$ & $0.20 \pm 0.05$ & $0.58 \pm 0.06$ & $0.54 \pm 0.08$ \\
\hline & matter & $\mathrm{Cc}_{\mathrm{b}}$ & $0.69 \pm 0.07$ & $0.64 \pm 0.10$ & $0.96 \pm 0.11$ & $0.94 \pm 0.18$ & $0.28 \pm 0.06$ & $0.33 \pm 0.09$ & $0.51 \pm 0.06$ & $0.53 \pm 0.11$ \\
\hline & & $\mathrm{CC}_{\mathrm{s}}$ & $0.80 \pm 0.06$ & $0.78 \pm 0.04$ & $1.32 \pm 0.16$ & $1.29 \pm 0.24^{\dagger}$ & $0.23 \pm 0.06$ & $0.25 \pm 0.04$ & $0.60 \pm 0.06$ & $0.60 \pm 0.09^{\dagger}$ \\
\hline & & ac & $0.79 \pm 0.07$ & $0.83 \pm 0.04$ & $1.18 \pm 0.20$ & $1.10 \pm 0.18$ & $0.21 \pm 0.07$ & $0.16 \pm 0.05$ & $0.54 \pm 0.07$ & $0.47 \pm 0.08$ \\
\hline & & ic & $0.74 \pm 0.05$ & $0.68 \pm 0.03^{\star \dagger}$ & $1.25 \pm 0.13$ & $1.08 \pm 0.19$ & $0.28 \pm 0.05$ & $0.30 \pm 0.04^{\dagger \dagger}$ & $0.60 \pm 0.06$ & $0.56 \pm 0.09^{\dagger}$ \\
\hline & & $a b$ & $0.75 \pm 0.07$ & $0.72 \pm 0.10$ & $1.40 \pm 0.14$ & $1.36 \pm 0.17$ & $0.30 \pm 0.06$ & $0.32 \pm 0.11$ & $0.67 \pm 0.04$ & $0.67 \pm 0.09$ \\
\hline & Gray & LD & $0.33 \pm 0.06$ & $0.37 \pm 0.05$ & $0.95 \pm 0.11$ & $0.94 \pm 0.30^{\dagger}$ & $0.58 \pm 0.06$ & $0.54 \pm 0.15$ & $0.70 \pm 0.07$ & $0.68 \pm 0.20^{\dagger}$ \\
\hline & matter & VPL-VPM & $0.28 \pm 0.05$ & $0.32 \pm 0.05$ & $0.93 \pm 0.08$ & $0.84 \pm 0.18$ & $0.62 \pm 0.05$ & $0.52 \pm 0.10^{\star}$ & $0.72 \pm 0.05$ & $0.63 \pm 0.12$ \\
\hline \multirow{8}{*}{ 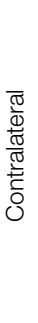 } & White & $\mathrm{Cc}_{\mathrm{g}}$ & $0.77 \pm 0.08$ & $0.80 \pm 0.03$ & $1.19 \pm 0.22$ & $1.19 \pm 0.20$ & $0.24 \pm 0.08$ & $0.20 \pm 0.03$ & $0.56 \pm 0.10$ & $0.53 \pm 0.08$ \\
\hline & matter & $\mathrm{cc}_{\mathrm{b}}$ & $0.66 \pm 0.10$ & $0.66 \pm 0.08$ & $0.91 \pm 0.17$ & $0.99 \pm 0.16$ & $0.29 \pm 0.10$ & $0.24 \pm 0.08$ & $0.49 \pm 0.12$ & $0.55 \pm 0.10$ \\
\hline & & $\mathrm{CC}_{\mathrm{S}}$ & $0.76 \pm 0.11$ & $0.76 \pm 0.03$ & $1.24 \pm 0.18$ & $1.23 \pm 0.22$ & $0.25 \pm 0.09$ & $0.25 \pm 0.04$ & $0.58 \pm 0.07$ & $0.57 \pm 0.09$ \\
\hline & & $\mathrm{ac}$ & $0.80 \pm 0.05$ & $0.83 \pm 0.05$ & $1.26 \pm 0.18$ & $1.08 \pm 0.22$ & $0.22 \pm 0.07$ & $0.17 \pm 0.07$ & $0.57 \pm 0.09$ & $0.47 \pm 0.12$ \\
\hline & & ic & $0.76 \pm 0.05$ & $0.74 \pm 0.04$ & $1.20 \pm 0.21$ & $1.06 \pm 0.19$ & $0.25 \pm 0.07$ & $0.24 \pm 0.04$ & $0.56 \pm 0.10$ & $0.51 \pm 0.08$ \\
\hline & & $a b$ & $0.77 \pm 0.06$ & $0.77 \pm 0.06$ & $1.39 \pm 0.19$ & $1.41 \pm 0.13$ & $0.27 \pm 0.06$ & $0.28 \pm 0.05$ & $0.65 \pm 0.07$ & $0.66 \pm 0.04$ \\
\hline & Gray & LD & $0.32 \pm 0.05$ & $0.32 \pm 0.05$ & $0.88 \pm 0.14$ & $0.79 \pm 0.22$ & $0.57 \pm 0.09$ & $0.49 \pm 0.13$ & $0.68 \pm 0.11$ & $0.60 \pm 0.16$ \\
\hline & matter & VPL-VPM & $0.30 \pm 0.03$ & $0.29 \pm 0.03$ & $0.90 \pm 0.11$ & $0.82 \pm 0.14$ & $0.58 \pm 0.07$ & $0.53 \pm 0.09$ & $0.69 \pm 0.09$ & $0.63 \pm 0.11$ \\
\hline
\end{tabular}

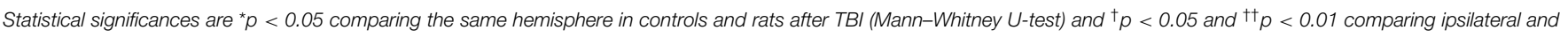
contralateral structures within the same animal (Wilcoxon test).

Abbreviations: ac, anterior commissure; ab, angular bundle; $c c_{b}$, body of the corpus callosum; $c c_{g}$, genu of corpus callosum; ic, internal capsule; cc s, splenium of corpus callosum; LD, laterodorsal thalamic nuclei; VPM, ventroposteromedial nucleus; VPL, ventroposterolateral nucleus.

\section{Histology from ex vivo DTI samples}

In histologic preparations, myelin loss was observed ipsilaterally and was moderate to severe (score 2 or 3 ) in all the animals (Table 2; Figures 2G,H, 3C,D). Contralateral myelin loss was also observed in all the rats, and was moderate to severe in 7 of 8 animals (Table 2). Varying amounts of iron accumulation were detected ipsilaterally in 6 of 8 rats with TBI and in 1 of 7 controls (Table 2).

\section{In vivo DTI}

DTI revealed a decrease in FA ipsilaterally in rats with TBI compared with controls ( $p<0.05$; Table 3). The decrease in FA was associated with an increase in $\mathrm{D}_{\perp}(p<0.05)$ and $\mathrm{MD}(p<0.05)$ ipsilaterally and contralaterally in rats after TBI compared with controls (Table 3). The differences between ex vivo and in vivo DTI data in this area might be due to the highly variable amounts of iron present in the tract (see Discussion).

\section{Histology from in vivo DTI samples}

As in ex vivo DTI samples, histologic preparations from in vivo DTI samples showed myelin loss in all the TBI rats as well as iron accumulation along this white matter structure. Similarly to ex vivo data, myelin loss and iron accumulation extended into the contralateral hemisphere, affecting the tissue microstructure (Table 3).

\section{Anterior Commissure}

There were no detectable abnormalities in DTI or histology (Tables 1-3).

\section{Internal Capsule}

\section{Ex vivo DTI}

FA was lower in TBI animals than in controls $(p<0.05)$, and lower ipsilaterally than contralaterally $(p<0.05)$ in TBI rats (Table 1). This was accompanied by an increase in $\mathrm{D}_{\perp}(p<0.01$ compared with the contralateral side; Table 1). MD was increased on the ipsilateral side compared with the contralateral side $(p<$ 0.05; Table 1).

\section{Histology from ex vivo DTI samples}

Myelin-stained sections showed myelin loss ipsilaterally in all the rats $(n=8)$ that was moderate to severe (score 2 or 3 ) in 5 of 8 rats (Table 2; Figures 2O,P). No iron accumulation was detected in this area (Table 2).

\section{In vivo DTI}

Similarly to ex vivo DTI, FA decreased after TBI when compared with controls ( $p<0.05$; Table 3). This decrease was accompanied by an increase in $\mathrm{D}_{\perp}(p<0.05$ compared to controls; Table 3).

\section{Histology from in vivo DTI samples}

Histologic preparations from the in vivo DTI samples confirmed the changes observed in the ex vivo group (Figure 4A).

\section{Angular Bundle \\ Ex vivo DTI}

None of the parameters differed from that in controls, ipsilaterally or contralaterally (Table 1; Figures 2I,J). Although FA and 
TABLE 2 | Semi-quantitative scores comparing controls and rats after TBI.

\begin{tabular}{|c|c|c|c|c|c|c|c|c|c|c|c|c|}
\hline \multicolumn{3}{|c|}{ Histology } & \multicolumn{2}{|c|}{ Myelin loss } & \multicolumn{2}{|c|}{ Iron accumulation } & \multicolumn{2}{|c|}{ Neurodegeneration } & \multicolumn{2}{|c|}{ Calcifications } & \multicolumn{2}{|c|}{ Total score } \\
\hline & & & Control & TBI & Control & TBI & Control & TBI & Control & TBI & Control & TBI \\
\hline \multirow{8}{*}{$\begin{array}{l}\frac{\bar{\sigma}}{\bar{d}} \\
\frac{\tilde{\sigma}}{\bar{\omega}} \\
\underline{\underline{0}}\end{array}$} & White & $\mathrm{CC}_{\mathrm{g}}$ & 0 & 0 & 0 & 0 & 0 & 0 & 0 & 0 & 0 & 0 \\
\hline & matter & $\mathrm{Cc}_{\mathrm{b}}$ & 0 & $2.0 \pm 0.8[2]$ & 0 & $0.6 \pm 1.0[0]$ & 0 & 0 & 0 & 0 & 0 & $2.6 \pm 1.7[2]$ \\
\hline & & $\mathrm{CC}_{\mathrm{s}}$ & 0 & $2.1 \pm 0.4[2]$ & $0.1 \pm 0.4[0]$ & $1.5 \pm 1.2[1.5]$ & 0 & 0 & 0 & 0 & $0.1 \pm 0.4[0]$ & $3.6 \pm 1.3[3.5]$ \\
\hline & & $\mathrm{ac}$ & 0 & 0 & 0 & 0 & 0 & 0 & 0 & 0 & 0 & 0 \\
\hline & & ic & 0 & $1.6 \pm 0.5[2]$ & 0 & 0 & 0 & 0 & 0 & 0 & 0 & $1.6 \pm 0.5[2]$ \\
\hline & & $a b$ & 0 & $2.6 \pm 0.5[3]$ & $0.1 \pm 0.4[0]$ & $0.3 \pm 0.5[0]$ & 0 & 0 & 0 & 0 & $0.1 \pm 0.4[0]$ & $2.9 \pm 0.6[3]$ \\
\hline & Gray & LD & 0 & $2.1 \pm 0.6[2]$ & 0 & 0 & 0 & $2.6 \pm 0.5[3]$ & 0 & $0.3 \pm 0.5[0]$ & 0 & $5.0 \pm 1.2[5.5]$ \\
\hline & matter & VPL-VPM & 0 & $1.8 \pm 0.5[2]$ & 0 & 0 & 0 & $2.5 \pm 0.5[2.5]$ & 0 & $2.0 \pm 1.1[2]$ & 0 & $6.3 \pm 1.9[6.5]$ \\
\hline \multirow{8}{*}{ 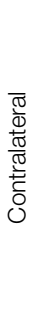 } & White & $\mathrm{CC}_{\mathrm{g}}$ & 0 & 0 & 0 & 0 & 0 & 0 & 0 & 0 & 0 & 0 \\
\hline & matter & $\mathrm{Cc}_{\mathrm{b}}$ & 0 & $1.1 \pm 0.6[1]$ & 0 & 0 & 0 & 0 & 0 & 0 & 0 & $1.1 \pm 0.6[1]$ \\
\hline & & $\mathrm{CC}_{\mathrm{s}}$ & 0 & $2.1 \pm 0.6[2]$ & 0 & 0 & 0 & 0 & 0 & 0 & 0 & $2.1 \pm 0.6[2]$ \\
\hline & & $\mathrm{ac}$ & 0 & 0 & 0 & 0 & 0 & 0 & 0 & 0 & 0 & 0 \\
\hline & & ic & 0 & 0 & 0 & 0 & 0 & 0 & 0 & 0 & 0 & 0 \\
\hline & & $a b$ & 0 & 0 & 0 & 0 & 0 & 0 & 0 & 0 & 0 & 0 \\
\hline & Gray & LD & 0 & $1.1 \pm 0.6[1]$ & 0 & 0 & 0 & 0 & 0 & 0 & 0 & $1.1 \pm 0.6[1]$ \\
\hline & matter & VPL-VPM & 0 & 0 & 0 & 0 & 0 & 0 & 0 & 0 & 0 & 0 \\
\hline
\end{tabular}

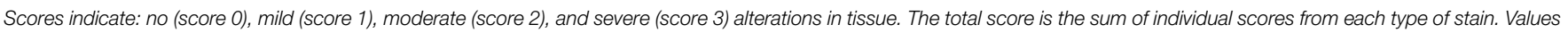
represent the mean value $\pm S D$ [median].

Abbreviations: ab, angular bundle; cc, corpus callosum; ic, internal capsule; scc, splenium of corpus callosum; LD, laterodorsal thalamic nuclei; VB, ventrobasal complex.

$\mathrm{D}_{\|}$appeared lower and $\mathrm{D}_{\perp}$ appeared elevated ipsilaterally in rats after TBI than in controls, the differences in these values did not reach statistical significance (Table 1).

\section{Histology from ex vivo DTI samples}

Histologic analysis revealed myelin loss ipsilaterally that was moderate to severe (score 2 or 3$)$ in all the rats $(n=8$; Table 2; Figures 2K,L). Iron accumulation was observed ipsilaterally in 2 of 8 rats after TBI and in 1 of 7 controls (Table 2).

\section{In vivo DTI}

In vivo DTI showed decreased FA in the ipsilateral side compared with the contralateral side $(p<0.05)$ in TBI rats (Table 3). Interestingly, FA was also decreased on the contralateral side in TBI rats compared with controls $(p<0.05$; Table 3$)$. $\mathrm{D}_{\|}$appeared lower on both the ipsilateral and contralateral sides compared with controls, although the difference was not statistically significant. $D_{\perp}$ was increased compared to that on the contralateral side ( $p<0.05$; Table 3$)$. The MD values remained unchanged.

\section{Histology from in vivo DTI samples}

Histologic preparations from in vivo DTI samples showed ipsilateral myelin loss as well as iron accumulation in all of the TBI rats. The cutting plane in the sections from the in vivo animal group allowed us to observe neighboring damage in the contralateral angular bundle (Figures 4C-E) that was difficult to visualize in the coronal sections of the ex vivo samples.

\section{Abnormalities in the Gray Matter after LFP Injury Laterodorsal Thalamic Nucleus \\ Ex vivo DTI}

$\mathrm{D}_{\|}$was increased compared with the contralateral side $(p<0.05$; Table 1), which was reflected as non-significant increasing trend in FA (Figures $\mathbf{2 M}, \mathbf{N}$ ). In TBI rats, MD was increased on the ipsilateral side compared with the contralateral side $(p<0.05$; Table 1).

\section{Histology from ex vivo DTI samples}

In histologic sections of TBI rats, myelin loss occurred ipsilaterally in all the rats $(n=8)$, and was moderate to severe (score 2 or 3 ) in 7 of 8 animals (Table 2; Figures 2O,P). The contralateral side also showed myelin loss in 7 of 8 rats, which was moderate to severe in 2 of 8 animals (Table 2). We also observed extensive neurodegeneration ipsilaterally that was moderate to severe in all the rats (Table 2; Figure 3E). Calcification was observed in 2 of 8 rats after TBI (Table 2; Figure 3F).

\section{In vivo DTI}

FA was significantly increased $(p<0.05)$ compared with the contralateral side in TBI rats (Table 3). Additionally, the contralateral side showed a decreased FA compared with control rats (Table 3). Ipsilaterally in TBI animals, $\mathrm{D}_{\|}$was increased compared with both controls $(p<0.05)$ and the contralateral side $\left(p<0.05\right.$; Table 3). $\mathrm{D}_{\perp}$ and $\mathrm{MD}$ were increased ipsilaterally compared with that in controls $(p<0.05)$ and contralaterally $(p<0.05$; Table 3). These two parameters were also increased on the contralateral side compared with control rats (Table 3 ). 
TABLE 3 | Fractional anisotropy (FA), and axial $\left(D_{||}\right)$, radial $\left(D_{\perp}\right)$, and mean (MD) diffusivities from ipsilateral and contralateral hemispheres in controls $(n=7)$ and rats after TBI $(n=8)$ obtained using in vivo DTI.

\begin{tabular}{|c|c|c|c|c|c|c|c|c|c|c|}
\hline \multicolumn{3}{|c|}{ In vivo } & \multicolumn{2}{|c|}{ FA } & \multicolumn{2}{|c|}{$D_{\|}\left(10^{-3} \mathrm{~mm}^{2} / \mathrm{s}\right)$} & \multicolumn{2}{|c|}{$D_{\perp}\left(10^{-3} \mathrm{~mm}^{2} / \mathrm{s}\right)$} & \multicolumn{2}{|c|}{$\mathrm{MD}\left(10^{-3} \mathrm{~mm}^{2} / \mathrm{s}\right)$} \\
\hline & & & Control & TBI & Control & TBI & Control & TBI & Control & TBI \\
\hline \multirow{8}{*}{ 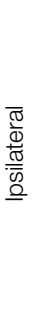 } & White matter & $\mathrm{CCg}_{\mathrm{g}}$ & $0.67 \pm 0.07$ & $0.78 \pm 0.08$ & $1.53 \pm 0.13$ & $1.57 \pm 0.21$ & $0.42 \pm 0.11$ & $0.31 \pm 0.10$ & $0.79 \pm 0.11$ & $0.73 \pm 0.11$ \\
\hline & & $\mathrm{cc}_{\mathrm{b}}$ & $0.65 \pm 0.06$ & $0.57 \pm 0.09$ & $1.43 \pm 0.14$ & $1.44 \pm 0.17$ & $0.49 \pm 0.10$ & $0.59 \pm 0.15$ & $0.80 \pm 0.10$ & $0.88 \pm 0.14$ \\
\hline & & $\mathrm{CC}_{\mathrm{s}}$ & $0.75 \pm 0.02$ & $0.58 \pm 0.10^{*}$ & $1.64 \pm 0.13$ & $1.54 \pm 0.10$ & $0.36 \pm 0.00$ & $0.61 \pm 0.10^{*}$ & $0.79 \pm 0.04$ & $0.92 \pm 0.06^{*}$ \\
\hline & & $\mathrm{ac}$ & $0.54 \pm 0.08$ & $0.64 \pm 0.09$ & $1.21 \pm 0.07$ & $1.35 \pm 0.17$ & $0.53 \pm 0.13$ & $0.45 \pm 0.13$ & $0.76 \pm 0.09$ & $0.75 \pm 0.14$ \\
\hline & & ic & $0.73 \pm 0.03$ & $0.61 \pm 0.04^{*}$ & $1.62 \pm 0.07$ & $1.55 \pm 0.07$ & $0.38 \pm 0.06$ & $0.53 \pm 0.08^{\star}$ & $0.80 \pm 0.06$ & $0.87 \pm 0.07$ \\
\hline & & $a b$ & $0.67 \pm 0.06$ & $0.57 \pm 0.04^{\dagger}$ & $1.80 \pm 0.18$ & $1.60 \pm 0.12$ & $0.50 \pm 0.04$ & $0.60 \pm 0.08^{\dagger}$ & $0.94 \pm 0.06$ & $0.93 \pm 0.09$ \\
\hline & Gray matter & LD & $0.32 \pm 0.01$ & $0.35 \pm 0.04^{\dagger}$ & $1.15 \pm 0.07$ & $1.59 \pm 0.10^{\star} \dagger$ & $0.70 \pm 0.04$ & $0.97 \pm 0.11^{\star \dagger}$ & $0.85 \pm 0.05$ & $1.17 \pm 0.10^{\star} \dagger$ \\
\hline & & VPL-VPM & $0.28 \pm 0.07$ & $0.32 \pm 0.03^{\dagger}$ & $1.08 \pm 0.11$ & $1.23 \pm 0.07^{\dagger}$ & $0.70 \pm 0.02$ & $0.76 \pm 0.06$ & $0.82 \pm 0.04$ & $0.92 \pm 0.06$ \\
\hline \multirow{8}{*}{ 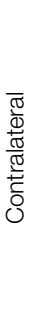 } & White matter & $\mathrm{CC}_{\mathrm{g}}$ & $0.71 \pm 0.09$ & $0.75 \pm 0.05$ & $1.55 \pm 0.14$ & $1.42 \pm 0.23$ & $0.41 \pm 0.16$ & $0.33 \pm 0.09$ & $0.79 \pm 0.15$ & $0.69 \pm 0.12$ \\
\hline & & $\mathrm{Cc}_{\mathrm{b}}$ & $0.64 \pm 0.03$ & $0.60 \pm 0.07$ & $1.46 \pm 0.09$ & $1.52 \pm 0.17$ & $0.50 \pm 0.04$ & $0.57 \pm 0.10$ & $0.82 \pm 0.05$ & $0.89 \pm 0.10$ \\
\hline & & $\mathrm{CC}_{\mathrm{s}}$ & $0.74 \pm 0.07$ & $0.60 \pm 0.05$ & $1.62 \pm 0.10$ & $1.61 \pm 0.07$ & $0.37 \pm 0.08$ & $0.58 \pm 0.05^{\star}$ & $0.79 \pm 0.05$ & $0.92 \pm 0.05^{\star}$ \\
\hline & & ac & $0.54 \pm 0.10$ & $0.63 \pm 0.06$ & $1.28 \pm 0.04$ & $1.27 \pm 0.29$ & $0.53 \pm 0.14$ & $0.43 \pm 0.10$ & $0.78 \pm 0.09$ & $0.71 \pm 0.16$ \\
\hline & & ic & $0.71 \pm 0.05$ & $0.64 \pm 0.05$ & $1.57 \pm 0.09$ & $1.63 \pm 0.04$ & $0.40 \pm 0.08$ & $0.52 \pm 0.06$ & $0.79 \pm 0.08$ & $0.89 \pm 0.04$ \\
\hline & & $a b$ & $0.71 \pm 0.05$ & $0.62 \pm 0.03^{\star}$ & $1.79 \pm 0.06$ & $1.66 \pm 0.11$ & $0.46 \pm 0.09$ & $0.54 \pm 0.05$ & $0.90 \pm 0.08$ & $0.91 \pm 0.07$ \\
\hline & Gray matter & LD & $0.36 \pm 0.02$ & $0.27 \pm 0.04^{*}$ & $1.15 \pm 0.04$ & $1.20 \pm 0.06$ & $0.67 \pm 0.02$ & $0.79 \pm 0.06^{\star}$ & $0.83 \pm 0.01$ & $0.93 \pm 0.05^{\star}$ \\
\hline & & VPL-VPM & $0.29 \pm 0.04$ & $0.28 \pm 0.03$ & $1.06 \pm 0.04$ & $1.06 \pm 0.10$ & $0.68 \pm 0.02$ & $0.68 \pm 0.02$ & $0.81 \pm 0.04$ & $0.81 \pm 0.04$ \\
\hline
\end{tabular}

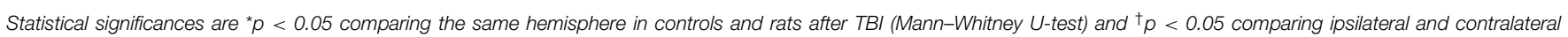
structures within the same animal (Wilcoxon test).

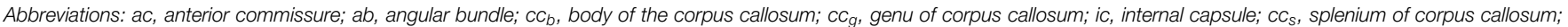
$L D$, laterodorsal thalamic nuclei; VPM, ventroposteromedial nucleus; VPL, ventroposterolateral nucleus.

\section{Histology from in vivo DTI samples}

Neurodegeneration, myelin loss, and calcification were detected ipsilaterally in rats after TBI, similarly to ex vivo DTI samples.

\section{VPM-VPL Nuclei Ex vivo DTI}

In the VPM-VPL nuclei, there was a decrease in $\mathrm{D}_{\perp}(p<0.05)$ ipsilaterally compared with controls (Table 1). In addition, there was a non-significant trend (slightly elevated FA and MD values on the ipsilateral side of TBI rats as well as lower $\mathrm{D}_{\|}$values ipsilaterally and contralalerally compared with controls) that was later confirmed by in vivo DTI (see below and Table 1).

\section{Histology from ex vivo DTI samples}

Histologic sections revealed myelin loss ipsilaterally in all the rats $(n=8)$, and it was moderate in severity (score 2$)$ in 6 of 8 animals (Table 2; Figures 2O,P). Extensive neurodegeneration that was moderate to severe (score 2 or 3 ) was also observed ipsilaterally in all the rats (Table 2; Figure 3E). Calcification was detected in all of the rats after TBI, which was severe (score 3 ) in 4 of 8 rats (Table 2; Figure 3F).

\section{In vivo DTI}

In in vivo experiments, FA was increased ipsilaterally $(p<$ $0.05)$ in association with an increase in $D_{\|}$, compared with the contralateral side $(p<0.05$; Table 3$)$.

\section{Histology from in vivo DTI samples}

Myelin loss was observed ipsilaterally in all the TBI rats compared with contralateral side, as in ex vivo DTI samples
(Figures 4A,B). Calcifications were also detected in most of the animals (Figures 4A,B). No iron accumulation was detected.

\section{Discussion}

The aim of the present study was to investigate the detection of secondary injury at a chronic time-point in a rat model of TBI using ex vivo and in vivo DTI, and to determine the underlying cellular changes based on histology. To our knowledge, this is the first study using DTI to investigate chronic alterations after LFP injury. Our analysis of ex vivo DTI data revealed persistent microstructural tissue changes in white matter tracts, such as the splenium of the corpus callosum, angular bundle, and internal capsule. Histologic examination revealed mainly a loss of myelinated axons and/or iron accumulation in the white matter tracts. Further, gray matter areas in the thalamus exhibited increased FA after TBI related to neurodegeneration, loss of myelinated fibers, and/or calcification in the chronic phase. Findings from in vivo DTI as well as examination of histologic preparations from in vivo DTI brains corroborated the findings and indicate the potential for using these changes as non-invasive markers of specific pathologic changes.

\section{DTI Detects Persistent Microstructural Alterations in the Chronic Phase after LFP Injury} Our results indicated that decreases in FA in white matter areas which is mainly associated with the loss of myelinated axons. This is consistent with findings from previous studies showing that FA is decreased in the white matter in a controlled cortical impact animal model of TBI (Mac Donald et al., 2007a,b), and in TBI 


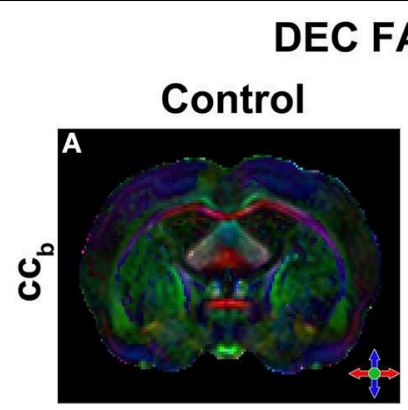

\section{A-maps}
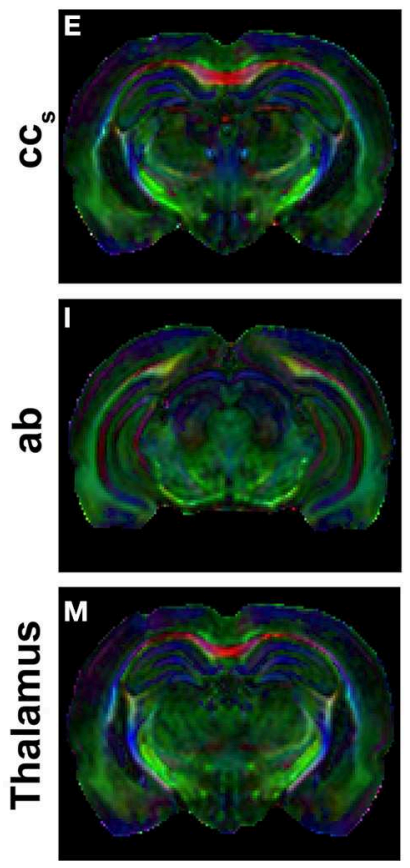

TBI
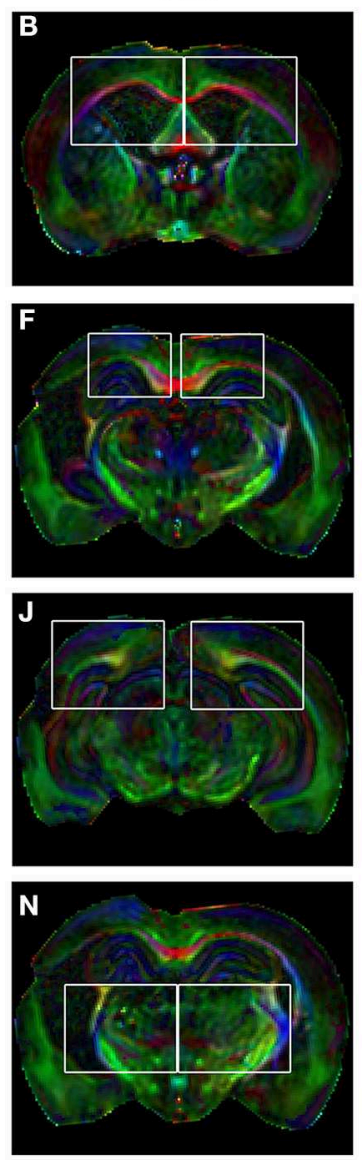

\section{Myelin staining}

Ipsilateral
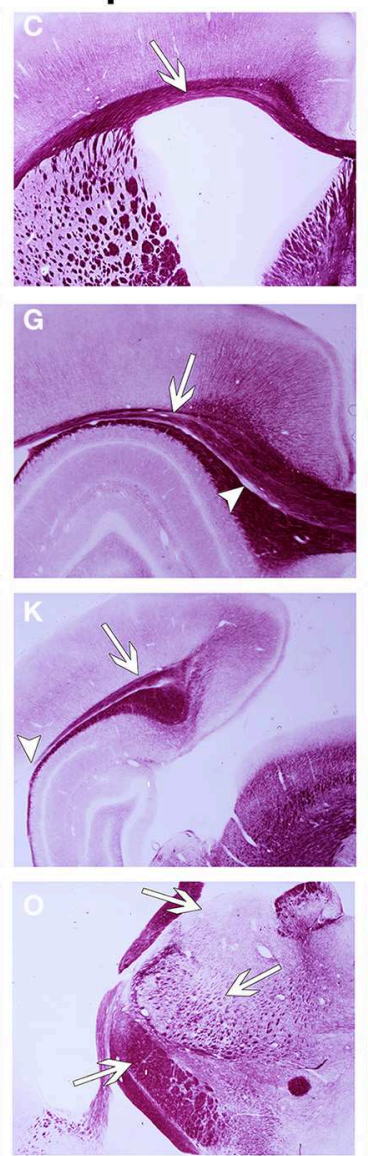

\section{Contralateral}
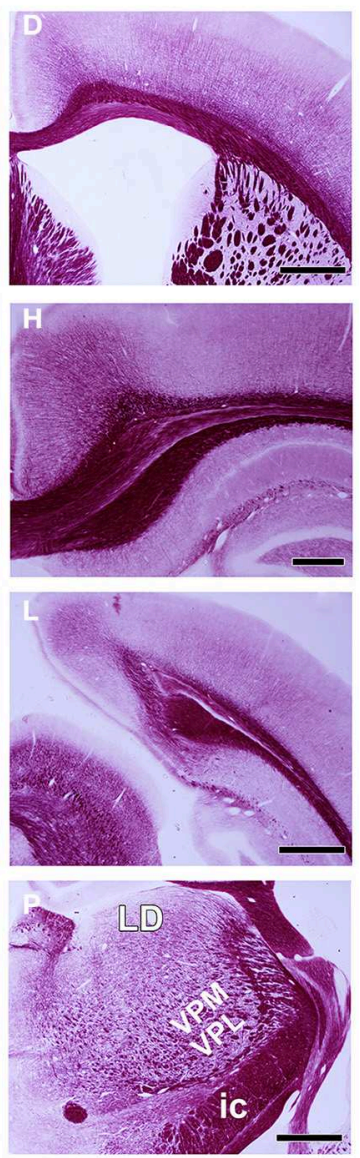

FIGURE 2 | Directionally encoded color (DEC) FA-maps of a sham-operated $(A, E, I, M)$ and a TBI $(B, F, J, N)$ animal. White squares indicate regions in the photomicrographs of myelin staining. Color coding in (A) red medial-lateral, green, rostral-caudal and blue dorsal-ventral. Representative photomicrographs of myelin-stained sections of the ipsilateral $\mathbf{( C , G , K , O )}$ and contralateral $\mathbf{( D , H , L , P )}$ hemisphere of a TBI animal. Panels (A-D) show the body of the corpus callosum. White arrow indicates the thinning of this structure.

Panels (E-H) show the splenium of the corpus callosum with thinning of the structure (white arrow) and loss of myelinated axons (white arrowhead). Panels (I-L) show the angular bundle. White arrow indicates the loss of myelinated axons and white arrowhead the disruption of this bundle. Panels (M-P) show the thalamus. In (G), the generalized cell loss in the thalamus is observed. White arrows indicate the loss of white matter in the laterodorsal thalamic nucleus, ventroposteromedial and ventroposterolateral nuclei and internal capsule. Abbreviations: $a b$, angular bundle; $c c_{b}$, corpus callosum; ic, internal capsule; $L D$, laterodorsal thalamic nucleus; $c c_{S}$, splenium of the corpus callosum; VPL-VPM, ventroposteromedial (VPM), and ventroposterolateral (VPL) nuclei. Scale bars: $1 \mathrm{~mm}$ (D,L,P) and $500 \mu \mathrm{m} \mathbf{( H )}$. patients (Sidaros et al., 2008). Massive axonal injury occurs in the corpus callosum in the acute phase after brain injury, slowing down after 4 days and ceasing within weeks (Mac Donald et al., 2007a,b). However, the body and splenium of the corpus callosum showed loss of axons in the contralateral hemisphere detected in the histology but not by DTI. Because of the continuity of the structure between both hemispheres, we expected that axonal loss would also occur contralaterally to the injury. In the body of the corpus callosum, milder changes were observed on the contralateral side (score 0 to 2 ) than on the ipsilateral side (score 1 to 3; Table 2). In the splenium of the corpus callosum, the severity of the axonal loss was similar on the ipsilateral side (score 2 to 3 ) and contralateral side (score 1 to 3; Table 2). We observed no iron accumulation on the contralateral side, however, in either the body or splenium of the corpus callosum. Although there was a similar loss of axons on the contralateral side, very little is known about the differences in microstructural and ultrastructural changes after injury to these areas as well as the limitations of the DTI detection. Further investigation is needed to study how differences in ultrastructure between the ipsilateral and contralateral hemispheres after brain may potentially influence the DTI contrast.

In white matter areas, the most consistent finding in eigenvalue-derived parameters was increased $\mathrm{D}_{\perp}$. This might be due to prolonged axonal degeneration (Song et al., 2002; Budde et al., 2011) and, together with decreased FA, may serve as a marker of an overall decrease in the amount of myelinated fibers. Interestingly, the changes observed in $\mathrm{D}_{\|}$were more variable. 

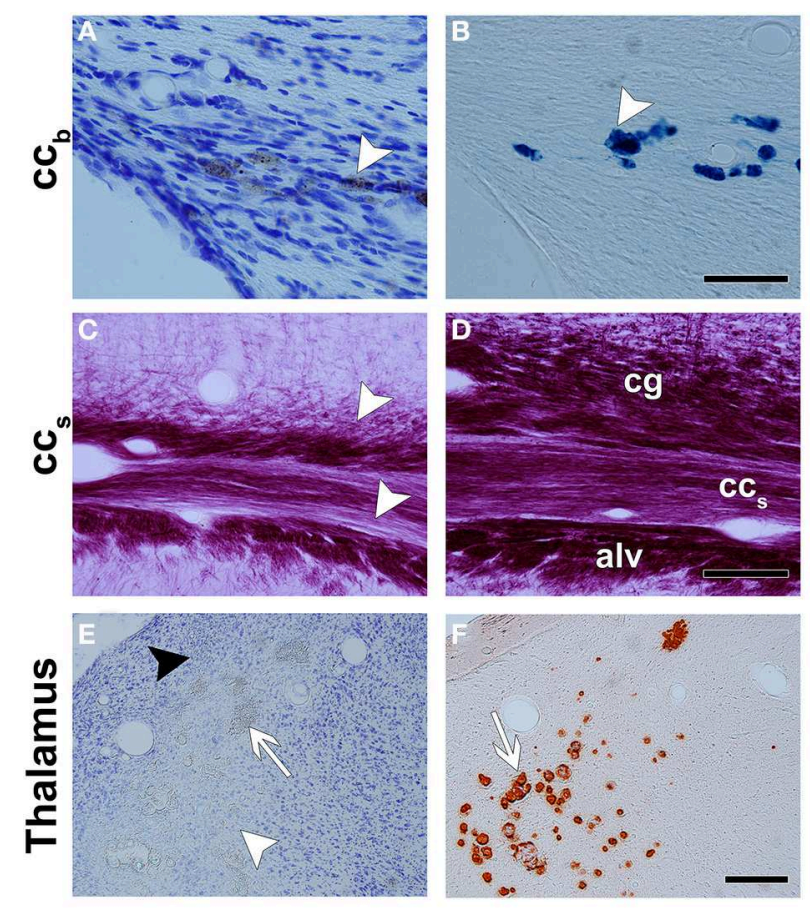

FIGURE 3 | Representative photomicrographs of Nissl- (A,E), Perl's(B), myelin- (C,D), and alizarin- (F) stained sections. The corpus callosum shows bleeding with a high iron content between the axons, which are dark in Nissl staining (white arrowhead in A) and intense blue in Perl's staining (white arrowhead in $\mathbf{B}$ ). (C,D) are photomicrographs of a myelin-stained section of the ipsilateral and contralateral hemispheres in a TBI animal, respectively. There is a loss of myelinated axons in the splenium of the corpus callosum and in adjacent areas, the cingulum and the alveus. In the thalamus, neurodegeneration (white arrowhead), gliosis (black arrowhead), and calcification (white arrow) are observed in Nissl-stained sections (E). Alizarin-stained sections show calcifications in intense red (white arrow in F). Abbreviations: $a / v$, alveus; $c c_{b}$, body of the corpus callosum; $c g$, cingulum; $c C_{S}$, splenium of the corpus callosum. Scale bars: $50 \mu \mathrm{m}$ (A,B), $100 \mu \mathrm{m}$ (C,D), and $300 \mu \mathrm{m}$ (E,F)

For example, in the splenium of the corpus callosum, $\mathrm{D}_{\|}$was increased, while in the internal capsule it tended to be lower. These differences between white matter tracts may be due to the morphology of the structures. The internal capsule appears to be more complex, as axons form a fan-shaped structure in contrast to the strictly parallel axonal organization in the corpus callosum and the angular bundle. The axons running through the internal capsule project to several cortical regions and are likely to undergo different changes depending on the severity of the cortical damage in the target areas. In this case, the detected changes in DTI metrics derived from a simple single tensor model have no straightforward interpretation, as the result is an average of changes caused by the damage processes to individual axons and is influenced by the orientation of these axons. In the future, more advanced diffusion MRI approaches, including high angular resolution diffusion imaging, with additional diffusion weighting directions, $b$-values, and advanced modeling, are likely to provide more comprehensive information regarding the microstructural changes in the tissue in these types of situations.
An alteration that may affect changes in the DTI parameters are the iron deposits found along the corpus callosum and the external capsule ipsilaterally to the trauma. Those deposits may come from hemorrhages or small bleeds in the acute phase that evolve to hemosiderin in the chronic phase. Iron deposits create local microscopic field gradients that may couple with diffusion field gradients and therefore locally influence diffusion metrics. This is also a likely explanation for some apparent inconsistencies in the data, such as different responses of $\mathrm{D}_{\|}$and $\mathrm{D}_{\perp}$ after $\mathrm{TBI}$ in ex vivo and in vivo DTI, in areas with high iron accumulation in the splenium of the corpus callosum.

In the thalamus, FA was increased in association with an increase in $\mathrm{D}_{\|}$and a decrease in $\mathrm{D}_{\perp}$. The changes observed in DTI co-localized with neurodegeneration, loss of myelinated axons, and calcification, as verified from the histology. Previous studies investigated the implications of diffuse axonal injury and neuronal atrophy in the ventrobasal complex after brain injury (Lifshitz et al., 2007). Cortical injuries may evoke anterograde/retrograde axonal degeneration within the thalamic nuclei. Therefore, neuronal death may occur in response to large, destructive cortical lesions (Ross and Ebner, 1990). More severe injuries provoke large acute axonal injuries, followed by neuronal atrophy, and consequently, neuronal death, inflammation, and accumulation of calcium (Lehto et al., 2012). It should be noted that similar to iron deposits in the corpus callosum, calcifications in the thalamus might influence DTI parameters by creating local magnetic fields.

\section{Chronic Tissue Damage after LFP Injury}

LFP injury is an established animal model of TBI (McIntosh et al., 1989) that mimics many of the features of human TBI, regarding both tissue damage and outcome (Graham et al., 2000; D’Ambrosio et al., 2004; Kharatishvili et al., 2007; Flygt et al., 2013; Gurkoff et al., 2013). The primary injury provokes a cortical lesion that, over time, progresses to distal areas in the brain. Chronic tissue alterations appear in the posterior part of the brain. A spatial gradient in the white matter (Mac Donald et al., 2007a), and the effect of the severity of the injury (Rutgers et al., 2008; Hylin et al., 2013) or position of the impact (Flygt et al., 2013) were previously investigated. Most studies using LFP injury focus on the acute and/or subacute phases, from a few hours to several days/weeks after injury (Graham et al., 2000; Flygt et al., 2013). The effect LFP injury on widespread myelin loss, axonal injury, inflammation, and changes in oligodendrocyte populations, as well as hemorrhages, have been extensively investigated in the acute and/or subacute phases (Dietrich et al., 1994; Flygt et al., 2013; Okubo et al., 2013). Rodriguez-Paez et al. (2005) investigated the progression of atrophy in the white and gray matter up to 1 year after LFP injury in rats. They demonstrated a progressive decrease in the number of axons in the fimbria, external capsule, thalamus, and cerebral cortex at several timepoints up to 1 year using light and electron microscopy. This result is consistent with the loss of myelinated axons detected in our histologic preparations. They also observed swollen axons and inflammation as many as 6 months after TBI, which is also consistent with the negative immunoreactivity against amyloid beta precursor protein (a marker for ongoing axonal injury) in 

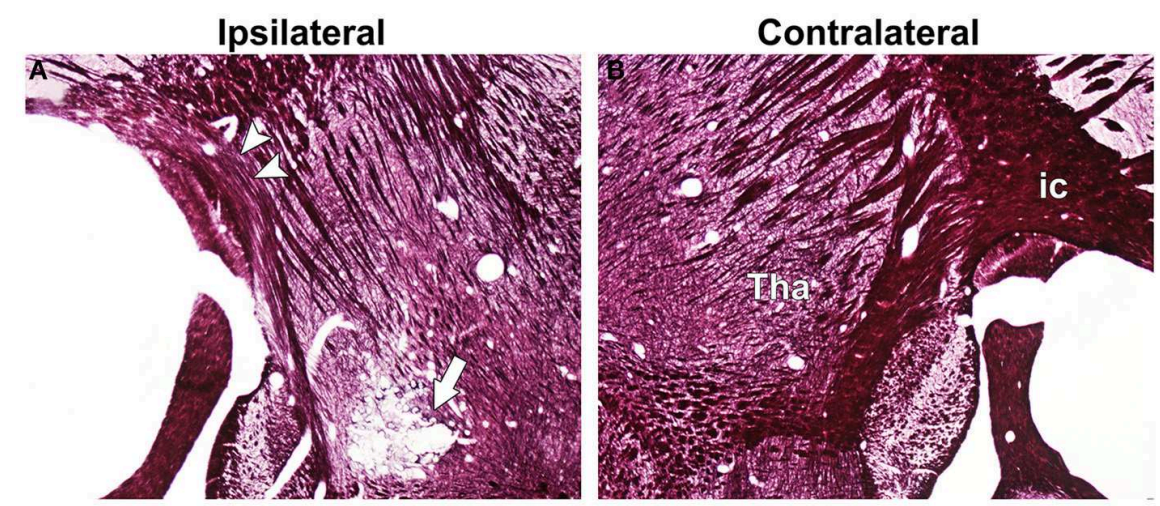

\section{Ipsilateral TB|}

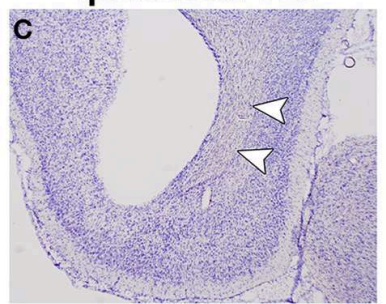

Contralateral TBI

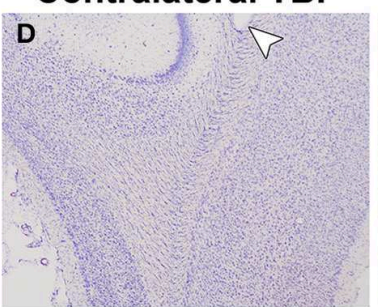

Contralateral control

E
FIGURE 4 | Representative photomicrographs of myelin-stained sections of the ipsilateral $(A)$ and contralateral $(B)$ hemispheres of a TBI animal from the in vivo group. Arrowheads point at the loss of myelinated axons in the internal capsule. The white arrow indicates calcifications in the thalamus. Representative photomicrographs of
Nissl-stained sections of ipsilateral (C) and contralateral (D) hemispheres of a TBI animal, and contralateral (E) hemisphere of a control animal from the in vivo group. Arrowheads indicate damage in the angular bundle ipsilaterally and contralaterally of a TBI rat compared with controls. Abbreviations: $a b$, angular bundle; ic, internal capsule; tha, thalamus. Scale bar: $500 \mu \mathrm{m}$. our sections (data not shown). Based on this, we conclude that our histologic observations are consistent with those of previous studies: the initial injury provokes axonal injury, neuronal damage, and hemorrhage, which progresses to a loss of myelinated axons, neuronal death, calcification, and the accumulation of hemosiderin with high iron content within the tissue several months after TBI.

\section{DTI as a Tool to Image TBI}

A key aspect of this study is the possibility of measuring microstructural changes in vivo with possible translation to human studies. Detection of statistically significant differences between group means in DTI parameters does not mean that these changes necessarily provide a biomarker for tissue damage or outcome measures after TBI, and this must be further explored in future studies with a larger number of animals and preferably a longitudinal study design. The finding that the in vivo DTI data were mostly consistent with ex vivo results, however, is encouraging. Previous studies revealed that FA and $\mathrm{D}_{\perp}$ changes in white matter are not significantly influenced by paraformaldehyde fixation, while $\mathrm{D}_{\|}$changes may be more difficult to detect in ex vivo preparations (Sun et al., 2003, 2005). Another possible confounding factor between in vivo and ex vivo results is the intravoxel incoherent motion due to the capillary blood pool. This could be a noteworthy factor, especially in the thalamus where significant vascular remodeling in chronic phase of TBI has been reported (Hayward et al., 2010).

\section{Conclusion}

Our results indicate that DTI is able to detect microstructural changes in the chronic phase of TBI. Detection of statistically significant differences between group means in DTI parameters does not necessarily indicate that these changes provide a biomarker for the tissue damage or outcome measures after TBI. This must be further investigated in in both white matter and gray matter in future studies with a larger number of animals and preferably a longitudinal study design. Whether DTI changes can serve as biomarkers for tissue damage or recovery after injury, however, requires further investigation. Experimental studies combining DTI and histologic approaches are extremely valuable for examining the origin of DTI changes at the cellular level. Overall, our data emphasize the potential of DTI as a tool to provide non-invasive information that aids in the assessment of comorbidities and alterations of the brain network in the chronic phase after brain injury.

\section{Acknowledgments}

This study was supported by the Academy of Finland (AS, OG, AP), Sigrid Juselius Foundation (AP), Finnish Cultural Foundation (AS), and UEF-Brain strategic funding from the University of Eastern Finland. We thank Ms. Maarit Pulkkinen for assistance with the histology. 


\section{References}

Aquino, C., Woolen, S., and Steenburg, S. D. (2015). Magnetic resonance imaging of traumatic brain injury: a pictorial review. Emerg. Radiol. 22, 65-78. doi: 10.1007/s10140-014-1226-0

Basser, P. J., Mattiello, J., and LeBihan, D. (1994). MR diffusion tensor spectroscopy and imaging. Biophys. J. 66, 259-267.

Basser, P. J., and Pierpaoli, C. (1996). Microstructural and physiological features of tissues elucidated by quantitative-diffusion-tensor MRI. J. Magn. Reson. B. 111, 209-219.

Basser, P. J., and Pierpaoli, C. (1998). A simplified method to measure the diffusion tensor from seven MR images. Magn. Reson. Med. 39, 928-934.

Budde, M. D., Janes, L., Gold, E., Turtzo, L. C., and Frank, J. A. (2011). The contribution of gliosis to diffusion tensor anisotropy and tractography following traumatic brain injury: validation in the rat using Fourier analysis of stained tissue sections. Brain 134, 2248-2260. doi: 10.1093/brain/awr161

D’Ambrosio, R., Fairbanks, J. P., Fender, J. S., Born, D. E., Doyle, D. L., and Miller, J. W. (2004). Post-traumatic epilepsy following fluid percussion injury in the rat. Brain 127, 304-314. doi: 10.1093/brain/awh038

Dietrich, W. D., Alonso, O., and Halley, M. (1994). Early microvascular and neuronal consequences of traumatic brain injury: a light and electron microscopic study in rats. J. Neurotrauma 11, 289-301.

Flygt, J., Djupsjö A., Lenne, F., and Marklund, N. (2013). Myelin loss and oligodendrocyte pathology in white matter tracts following traumatic brain injury in the rat. Eur. J. Neurosci. 38, 2153-2165. doi: 10.1111/ejn.12179

Gallagher, C. N., Hutchinson, P. J., and Pickard, J. D. (2007). Neuroimaging in trauma. Curr. Opin. Neurol. 20, 403-409. doi: 10.1097/WCO.0b013e32821b987b

Graham, D. I., Raghupathi, R., Saatman, K. E., Meaney, D., and McIntosh, T. K. (2000). Tissue tears in the white matter after lateral fluid percussion brain injury in the rat: relevance to human brain injury. Acta Neuropathol. 99, 117-124. doi: 10.1007/PL00007414

Gurkoff, G. G., Gahan, J. D., Ghiasvand, R. T., Hunsaker, M. R., Van, K., Feng, J. F., et al. (2013). Evaluation of metric, topological, and temporal ordering memory tasks after lateral fluid percussion injury. J. Neurotrauma 30, 292-300. doi: $10.1089 /$ neu. 2012.2463

Hayward, N. M., Immonen, R., Tuunanen, P. I., Ndode-Ekane, X. E., Gröhn, O., and Pitkänen, A. (2010). Association of chronic vascular changes with functional outcome after traumatic brain injury in rats. J. Neurotrauma 27, 2203-2219. doi: 10.1089/neu.2010.1448

Hyder, A. A., Wunderlich, C. A., Puvanachandra, P., Gururaj, G., and Kobusingye, O. C. (2007). The impact of traumatic brain injuries: a global perspective. NeuroRehabilitation. 22, 341-353.

Hylin, M. J., Orsi, S. A., Zhao, J., Bockhorst, K., Perez, A., Moore, A. N., et al. (2013). Behavioral and histopathological alterations resulting from mild fluid percussion injury. J. Neurotrauma 30, 702-715. doi: 10.1089/neu.2012. 2630

Jenkinson, M., Bannister, P., Brady, M., and Smith, S. (2002). Improved optimization for the robust and accurate linear registration and motion correction of brain images. Neuroimage 17, 825-841. doi: 10.1006/nimg.2002.1132

Jenkinson, M., and Smith, S. (2001). A global optimisation method for robust affine registration of brain images. Med. Image Anal. 5, 143-156. doi: 10.1016/S13618415(01)00036-6

Kharatishvili, I., Immonen, R., Gröhn, O., and Pitkänen, A. (2007). Quantitative diffusion MRI of hippocampus as a surrogate marker for post-traumatic epileptogenesis. Brain 130, 3155-3168. doi: 10.1093/brain/awm268

Kharatishvili, I., Nissinen, J. P., McIntosh, T. K., and Pitkänen, A. (2006). A model of posttraumatic epilepsy induced by lateral fluid-percussion brain injury in rats. Neuroscience 140, 685-697. doi: 10.1016/j.neuroscience.2006. 03.012

Kinnunen, K. M., Greenwood, R., Powell, J. H., Leech, R., Hawkins, P. C., Bonnelle, V., et al. (2011). White matter damage and cognitive impairment after traumatic brain injury. Brain 134, 449-463. doi: 10.1093/brain/ awq347

Kumar, R., Husain, M., Gupta, R. K., Hasan, K. M., Haris, M., Agarwal, A. K., et al. (2009). Serial changes in the white matter diffusion tensor imaging metrics in moderate traumatic brain injury and correlation with neuro-cognitive function. J. Neurotrauma 26, 481-495. doi: 10.1089/neu.2008.0461
Laitinen, T., Sierra, A., Pitkänen, A., and Gröhn, O. (2010). Diffusion tensor MRI of axonal plasticity in the rat hippocampus. Neuroimage 51, 521-530. doi: 10.1016/j.neuroimage.2010.02.077

Lehto, L. J., Sierra, A., Corum, C. A., Zhang, J., Idiyatullin, D., Pitkänen, A., et al. (2012). Detection of calcifications in vivo and ex vivo after brain injury in rat using SWIFT. Neuroimage 61, 761-772. doi: 10.1016/j.neuroimage.2012. 03.002

Lifshitz, J., Kelley, B. J., and Povlishock, J. T. (2007). Perisomatic thalamic axotomy after diffuse traumatic brain injury is associated with atrophy rather than cell death. J. Neuropathol. Exp. Neurol. 66, 218-229. doi: 10.1097/01.jnen.0000248558.75950.4d

Maas, A. I., Stocchetti, N., and Bullock, R. (2008). Moderate and severe traumatic brain injury in adults. Lancet Neurol. 7, 728-741. doi: 10.1016/S14744422(08)70164-9

Mac Donald, C. L., Dikranian, K., Bayly, P., Holtzman, D., and Brody, D. (2007b). Diffusion tensor imaging reliably detects experimental traumatic axonal injury and indicates approximate time of injury. J. Neurosci. 27, 11869-11176. doi: 10.1523/JNEUROSCI.3647-07.2007

Mac Donald, C. L., Dikranian, K., Song, S. K., Bayly, P. V., Holtzman, D. M., and Brody, D. L. (2007a). Detection of traumatic axonal injury with diffusion tensor imaging in a mouse model of traumatic brain injury. Exp. Neurol. 205, 116-131. doi: 10.1016/j.expneurol.2007.01.035

Mäkinen, S., van Groen, T., Clarke, J., Thornell, A., Corbett, D., Hiltunen, M., et al. (2008). Coaccumulation of calcium and beta-amyloid in the thalamus after transient middle cerebral artery occlusion in rats. J. Cereb. Blood Flow Metab. 28, 263-268. doi: 10.1038/sj.jcbfm.9600529

Marshall, L. F., Eisenberg, H., Jane, J. A., Marshall, S. B., and Klauber, M. R. (1991). A new classification of head injury based on computerized tomography. J. Neurosurg. 75, S14-S20.

McIntosh, T. K., Vink, R., Noble, L., Yamakami, I., Fernyak, S., Soares, H., et al. (1989). Traumatic brain injury in the rat: characterization of a lateral fluid-percussion model. Neuroscience 28, 233-244.

Mori, S., and Zhang, J. (2006). Principles of diffusion tensor imaging and its applications to basic neuroscience research. Neuron 51, 527-539. doi: 10.1016/j.neuron.2006.08.012

Okubo, S., Xi, G., Keep, R. F., Muraszko, K. M., and Hua, Y. (2013). Cerebral hemorrhage, brain edema, and heme oxygenase-1 expression after experimental traumatic brain injury. Acta Neurochir. 118, 83-87. doi: 10.1007/978-3-70911434-6_14

Pajevic, S., and Pierpaoli, C. (1999). Color schemes to represent the orientation of anisotropic tissues from diffusion tensor data: application to white matter fiber tract mapping in the human brain. Magn. Reson. Med. 42, 526-540.

Rodriguez-Paez, A. C., Brunschwig, J. P., and Bramlett, H. M. (2005). Light and electron microscopic assessment of progressive atrophy following moderate traumatic brain injury in the rat. Acta Neuropathol. 109, 603-616. doi: 10.1007/s00401-005-1010-z

Ross, D. T., and Ebner, F. F. (1990). Thalamic retrograde degeneration following cortical injury: an excitotoxic process? Neuroscience 35, 525-550.

Rutgers, D. R., Toulgoat, F., Cazejust, J., Fillard, P., Lasjaunias, P., and Ducreux, D. (2008). White matter abnormalities in mild traumatic brain injury: a diffusion tensor imaging study. AJNR Am. J. Neuroradiol. 29, 514-519. doi: 10.3174/ajnr.A0856

Savander, V., Go, C. G., Ledoux, J. E., and Pitkänen, A. (1996). Intrinsic connections of the rat amygdaloid complex: projections originating in the accessory basal nucleus. J. Comp. Neurol. 374, 291-313.

Sidaros, A., Engberg, A. W., Sidaros, K., Liptrot, M. G., Herning, M., Petersen, P., et al. (2008). Diffusion tensor imaging during recovery from severe traumatic brain injury and relation to clinical outcome: a longitudinal study. Brain 131, 559-572. doi: 10.1093/brain/awm294

Sloviter, R. S. (1982). A simplified Timm stain procedure compatible with formaldehyde fixation and routine paraffin embedding of rat brain. Brain Res. Bull. 8, 771-774.

Song, S. K., Sun, S. W., Ramsbottom, M. J., Chang, C., Russell, J., and Cross, A. H. (2002). Dysmyelination revealed through MRI as increased radial (but unchanged axial) diffusion of water. Neuroimage 17, 1429-1436. doi: 10.1006/nimg.2002.1267

Sun, S. W., Neil, J. J., Liang, H. F., He, Y. Y., Schmidt, R. E., Hsu, C. Y., et al. (2005). Formalin fixation alters water diffusion coefficient magnitude 
but not anisotropy in infarcted brain. Magn. Reson. Med. 53, 1447-1451. doi: 10.1002/mrm. 20488

Sun, S. W., Neil, J. J., and Song, S. K. (2003). Relative indices of water diffusion anisotropy are equivalent in live and formalin-fixed mouse brains. Magn. Reson. Med. 50, 743-748. doi: 10.1002/mrm. 10605

van der Zwaag, W., Marques, J. P., Lei, H., Just, N., Kober, T., and Gruetter, R. (2009). Minimization of Nyquist ghosting for echo-planar imaging at ultra-high fields based on a "negative readout gradient" strategy. J. Magn. Reson. Imaging 30, 1171-1178. doi: 10.1002/jmri.21951
Conflict of Interest Statement: The authors declare that the research was conducted in the absence of any commercial or financial relationships that could be construed as a potential conflict of interest.

Copyright (c) 2015 Laitinen, Sierra, Bolkvadze, Pitkänen and Gröhn. This is an openaccess article distributed under the terms of the Creative Commons Attribution License (CC BY). The use, distribution or reproduction in other forums is permitted, provided the original author(s) or licensor are credited and that the original publication in this journal is cited, in accordance with accepted academic practice. No use, distribution or reproduction is permitted which does not comply with these terms. 